\title{
Novel Design of an Intrauterine Insemination Cannula
}

\section{Ritvik Vasan}

\begin{abstract}
Intrauterine insemination (IUI) is a method of assisted conception that involves transfer of fast moving sperms using an IUI cannula into the uterine cavity at the time of anticipated (or expected) ovulation, thereby increasing chance of pregnancy.

Existing cannulas are designed to be easy to use, harmless to the cervix/endometrium, capable of negotiating the cervical cavity, and to avoid the reflux of inseminate fluid. Some of the factors which influence success of pregnancy are actual number of motile sperms, number of sperms with normal morphology, volume of inseminate and sterility during procedure. This paper proposes a novel 'completely closed loop' design for an IUI cannula that ensures complete emptying of washed sperm during insemination, leaving no dead space while ensuring sterility during procedure.
\end{abstract}

Keywords: Intrauterine insemination, Cannula, Insemination, Catheter, Stillet.

How to cite this article: Vasan R. Novel Design of an Intrauterine Insemination Cannula. Int J Infertil Fetal Med 2015;6(1): 15-19.

Source of support: This work was supported by Manipal Ankur, Bengaluru, India.

Conflict of interest: None

Date of received: 08-01-15

Date of acceptance: 10-02-15

Date of publication: April 2015

\section{INTRODUCTION}

Intrauterine insemination (IUI) is a common first-line treatment prescribed by many andrologists, gynecologists and reproductive endocrinologists. Many couples undergo this procedure each year, as a treatment for male and/or female subfertility.

Sperm processing or washing involves a laboratory procedure to separate fast moving sperm from more sluggish or nonmoving sperm. An IUI is the done to place the washed sperm into the woman's uterine cavity, close to the time of ovulation (when the egg is released from

\section{Research Assistant}

Department of Manipal Ankur Andrology and Reproductive Services, Innovation Lab, Manipal Ankur, Bengaluru Karnataka, India

Corresponding Author: Ritvik Vasan, Research Assistant Department of Manipal Ankur Andrology and Reproductive Services, Innovation Lab, Manipal Ankur, Bengaluru, Karnataka India, Phone: 9561295493, e-mail: ritvikvasan@gmail.com the ovary in the middle of the monthly cycle). ${ }^{1}$ Usually, 3 to 6 cycles of IUI is advised before moving on to in vitro fertilization (IVF). ${ }^{2}$ This is because IUI costs only a fraction of IVF and is less invasive. ${ }^{3}$ A recent Cochrane review has recommended the use of IUI in patients with unexplained infertility as the success rates are similar to those achieved with IVF/ICSI in this patient population. ${ }^{4}$ The average success rate for pregnancy in women in the age group below 35 years is $15.8 \%$, and it decreases steadily with advancing age, to about $5 \%$ at 40 years and is virtually 0 at above 44 years. ${ }^{5}$

From a scientific point of view, controversy still surrounds the effectiveness of this very popular treatment procedure. This may be explained by the fact that most studies are retrospective and not only vary in the comparison of the study group but also in the use or non-use of different ovarian super-ovulation regimen, the number of inseminations per treatment cycle, different methods of timing ovulation, different sites of insemination, various methods of sperm preparation and the well or not use of additives, such as antioxidants, platelet-activating factor (PAF), etc. ${ }^{6}$

The most important selection criterion for suitability of couples for treatment by IUI is the sperm quality and sperm number. Few reports have shown an evident correlation between progressive motility in whole sperm and pregnancy rate (PR). ${ }^{7-12}$ Progressive motility has significant influence on the ongoing pregnancy rate at $11.5 \%$ for the over or equal to 10 vs $0 \%$ for the under $10 \%(\mathrm{p}<0.017)$. The clinical PR per couple decreased from 12.5 to $0 \%$, if teratozoospermia was higher than the abnormal form threshold $(>80 \% ; \mathrm{p}<0.0001)$. Regarding the total motile spermatozoa inseminated, the clinical PR per couple was $4.6 \%$ when total motile sperm (TMS) was lower than 5 million and was significantly higher $(11.8 \%)$ when this number was above 5 million $(p=0.03){ }^{13}$ This has also been confirmed by Haim et $\mathrm{al}^{11}$ who found a threshold of $10 \%$ as a predictive value of IUI outcome.

Intrauterine insemination cannula plays a crucial role of transferring the inseminate fluid to facilitate fertilization. The device needs to have the following characteristics which positively influence the clinical PR:

- Accurate delivery of actual number of motile sperms

- Number of sperms with normal morphology

- Volume of the inseminate

- Sterility during the procedure. 


\section{Role of the IUI Cannula}

The basis of IUI procedure involves the use a catheter to deliver washed spermatozoa past the cervical mucus barrier into the uterine cavity, and thereby, it increases the sperm concentration at the site of fertilization. Even so, the pregnancy rate per IUI cycle has been shown to vary markedly. ${ }^{14}$ Therefore, traditionally, couples have been advised to undertake three to six IUI cycles before considering IVF. ${ }^{2}$

One aspect of assisted reproduction that has gained importance over the past few years is catheter technology. In a recently published systematic review and metaanalysis, the softness of the transfer cannula was found to be a determining factor in the success of the procedure. ${ }^{15}$ The explanation for this is theorized to be associated with either the traumatic effects of the catheter during introduction into the uterine cavity or junctional zone contractions with the firmer catheters. ${ }^{16,17}$ Irrespective of the etiology, type of the transfer cannula has proved to have a considerable effect on treatment outcome.

Proctor and Boone ${ }^{18}$ have recently shown that use of a flexible catheter is associated with a higher PR, although it costs more than the rigid catheter. Flexible catheter decreases the cost of IUI treatment as fewer cycles are needed.

\section{Cannula Design}

Effective sperm delivery into the uterine cavity is the most critical aspect of the catheter function. A review of existing literature was done to study the various aspects of catheter design. Sperm delivery is the most critical event and any reduction in the efficiency of catheter would compromise the success rate of the procedure.

Catheters vary in length, caliber and location of the distal port (end or side-dispersion systems). They also differ in the degree of firmness and rigidity. In general, there are some technical aspects that are similar to all insemination catheters. ${ }^{19}$

- Ease of use

- Firm enough to negotiate the curvature of the cervix

- Soft to avoid any trauma to the endocervix and/or endometrium

- Tip of the cannula should occupy a small volume

- Design should avoid reflux of inseminated material. Components of design of cannula include: flexibility, single/double lumen, inherent memory, material used, terminal round tip or lateral tips, and the length of the cannula.

\section{Soft Artificial Insemination Cannula}

The Wallace ${ }^{\mathrm{TM}}$ artificial insemination catheter (Smiths Medical) is a soft, flexible, double-lumen catheter, utilizing a coaxial system. Inner catheter is $18 \mathrm{~cm}$ long, with a rounded tip. It utilizes a bilateral side dispersion design to help prevent contamination and blockage of the catheter during insertion. Its outer sheath is flexible, but with memory, which can be pre-shaped to aid insertion.

The Cook ${ }^{\mathrm{TM}}$ Soft-Pass ${ }^{\circledR}$ artificial insemination catheter (Cook Women'sHealth, Spencer, IN USA) is soft, flexible, double-lumen, coaxial catheter system. Inner catheter is $19 \mathrm{~cm}$ long with a rounded tip. Its outer guide catheter is similar to the outer sheath of the Wallace artificial insemination catheter.

The Gynetics ${ }^{\mathrm{TM}}$ artificial insemination catheter (Gynetics Medical Products, Hamont-Achel, Belgium) also has a soft, flexible, double-lumen, coaxial catheter system. It is $20.6 \mathrm{~cm}$ in length with a rounded tip. Similar to the Wallace artificial insemination catheter, it uses two lateral ports at the distal end for intrauterine sperm distribution. It also comes with a solid, thicker outer catheter for bypassing the difficult cervix.

\section{Firm Artificial Insemination Cannula}

Firm cannulas are generally not preferred, except in difficult situations, as there is a higher chance of damage to the cervix during insemination. The Tomcat ${ }^{\mathrm{TM}}$ artificial insemination catheter (Kendall Sovereign, Mansfield, MA, USA) is a firm, semi-rigid, single-lumen catheter. It is $11.4 \mathrm{~cm}$ long, 3.5 French ( $\mathrm{Fr}$ ) and with an open-end design. With its inherent memory, its design enables the catheter to be molded to the curvature of the uterus.

The Makler ${ }^{\mathrm{TM}}$ IUI Cannula and Insemination Device (Sefi-Medical Instruments, Haifa, Israel) is composed of two main parts: (i) a cannula that can be fitted to the tip of $1.0 \mathrm{ml}$ tuberculin syringe, and (ii) a carrier to hold the syringe. The catheter is a firm, semi-rigid, single-lumen catheter with a rounded tip. It has a flared-base shape, which serves to seal the cervix at the time of insemination to help prevent the backflow of the injected contents. This is accomplished by entirely obliterating the cervical external os by the flared base of the cannula. The clamp is then fixed to the outer brim of the speculum and the tension of the spring is adjusted so that the cannula is gently pressed against the cervical outlet.

We have evaluated the various components of the catheter design and hypothesized that the present cannula design has a flaw in not eliminating the dead space, and there could be situations in which sperm may not be effectively transferred into the uterine cavity. In the existing cannula, dead space is eliminated by the clinician using atmospheric air as a conduit to fill the cannula, and use it as a medium to push the seminal plasma. This method makes the process inefficient due to possibility of contamination and infection, leading to 
reduction in pregnancy rates. Eliminating dead space is primarily needed as nulliparous uterine luminal volume is about $0.3 \mathrm{ml}$ and pushing a larger volume would lead to spilling of the inseminate fluid.

\section{NEW DESIGN DEVELOPMENT}

\section{Rationale}

A preliminary study was done taking $0.5 \mathrm{ml}$ volume of inseminate and $1 \mathrm{ml}$ volume of inseminate fluid using a standard IUI cannula and it was found post-insemination 0.2 to $0.3 \mathrm{ml}$ of fluid could be potentially left behind in an IUI cannula, if the clinician had not used air as a medium to wash the cannula after pushing the seminal fluid. This was seen uniformly across all the cannulas listed above. As doctors using IUI cannula have different techniques of insemination and as the process requires complete emptying of sperm sample into the uterus with no contamination during the procedure, a closed system of IUI cannula was envisaged.

\section{Design Principles}

1. To avoid contamination with environmental air during the procedure of IUI making it a completely closed system.

2. To ensure there is complete emptying of the seminal fluid from the IUI cannula during insemination, thereby eliminating dead space.

An image of the new IUI cannula is shown in Figure 1, along with the dimensions of its various parts. The cannula has an inner soft portion made up of low-density polymer and the outer portion made up of polypropylene (PP). The side channel for fixing the syringe and for introducing the stillet is made up of high-density polypropylene. The cannula has a softer part situated distally for negotiating the cervix, and a stiffer proximal part for introduction through the vaginal speculum. The

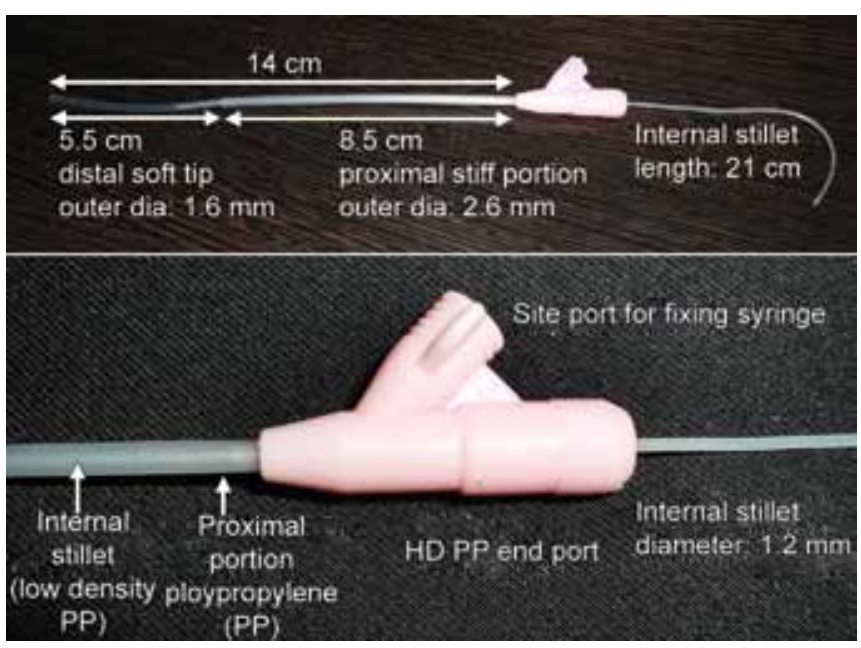

Fig. 1: New design for intrauterine insemination cannula terminal end port is provided for introducing the stillet straight on and a side port about $1 \mathrm{~cm}$ distal to the end port for fixing a syringe to push the seminal fluid. An opening is provided for the release of sperm in to the uterine cavity. All of the materials used are nontoxic and human grade materials.

\section{Existing Devices and the New Design Differentiation}

- All IUI cannulas are used horizontally or in fact, the tip curves upwards in the uterus.

- Seminal plasma with sperm which is inseminated has a higher viscosity than water.

- A syringe or a tuberculin syringe $(1 \mathrm{ml})$ is used to load sperm after preparation.

- The volume used to inseminate is 0.5 to $1 \mathrm{ml}$.

- Sperm is highly concentrated.

- Sperm having higher density will, more likely, settle at the bottom of the syringe.

- In effect, with a $1 \mathrm{ml}$ syringe, $0.2 \mathrm{ml}$ of seminal fluid stays in the IUI cannula as air is displaced by liquid. This amounts to $20 \%$ reduction in the volume of the inseminate.

- Some gynecologists are aware of this, and use air in the end to re-displace this fluid into the uterus. Injection of air, however minimal, carries the risk of infection, negating the chances of pregnancy.

The new IUI cannula eliminates the need to injection of air with its new design. Terminal portion of the cannula is soft with end opening, along with a stiffer proximal part with a stillet/proximal port and a side port for insemination.

\section{Insemination Procedure}

Sperm is aspirated using a syringe from a side port as shown in Figure 1. Volume of inseminate in soft distal portion is $0.249 \mathrm{ml}$ and volume of inseminate in stiffer proximal portion is 0.384 . Thus, the total volume of inseminate in the entire catheter is $0.633 \mathrm{ml}$. The cannula is then placed in appropriate position in the uterus and the syringe is compressed, pushing the semen out. Since a small volume $(0.16 \mathrm{ml})$ of semen sample is left behind, the stillet is pushed into the cannula through adjacent port, thereby ensuring complete emptying of semen. Additionally, the procedure utilizes no atmospheric air, ensuring sterility. This ensures the system is a closed system.

\section{EVALUATION OF THE NEW IUI CANNULA}

New design of the IUI cannula was evaluated by repeated measurements of volume of fluid. The volumes transferred by currently available cannula are shown in Table 1, for comparison. 
Table 1: Dimensions of existing cannula

\begin{tabular}{llllll}
\hline & $\begin{array}{l}\text { External } \\
\text { diameter } \\
\text { Model }\end{array}$ & $\begin{array}{l}\text { Internal } \\
\text { diameter } \\
(\mathrm{cm})\end{array}$ & $\begin{array}{l}\text { Length } \\
(\mathrm{cm})\end{array}$ & $\begin{array}{l}\text { Reported } \\
\text { volume } \\
(\mathrm{ml})\end{array}$ & $\begin{array}{l}\text { Actual } \\
\text { volume } \\
(\mathrm{ml})\end{array}$ \\
\hline IUI-S & 0.21 & 0.165 & 17 & 0.40 & 0.22 \\
IUI-G & 0.16 & 0.065 & 17 & 0.10 & 0.28 \\
Gynetics & 0.18 & - & 20.6 & - & 0.55 \\
Shepard & 0.54 & - & 20 & - & 4.57 \\
\hline
\end{tabular}

Devon 6F/8F catheter: Maximum length $17.3 \mathrm{~cm}$

A prospective study of the performance of the new cannula in IUI procedure has been conducted to evaluate the new design. The study consisted of 90 patients undergoing IUI-H treatment cycles at the Manipal Ankur Andrology and Reproductive Services Innovation Lab, Bengalure, India, from April 2012 to December 2014. Patients were classified into two groups on the basis of the severity of the male or female factor, seminal parameters/trial processing parameters which were graded on severity along with medication history. Both the groups had standard stimulation protocols with clomiphene citrate (CC) and/or gonadotropin followed by a single or double IUI depending on follicular rupture each cycle. Both groups had an insemination volume of $0.8 \mathrm{ml}$, concentration above 40 million with post wash motility of $90 \%$ and above. A standard insemination cannula was used for the first group (control group) and the new IUI cannula was used for the other group (study group). The main outcome measure was the clinical pregnancy rate and the volume of inseminate/sperms leftover in the cannula. A comparative statistical analysis was performed using t-test. The results of the preliminary study are shown in Table 2.

\section{CONCLUSION}

Today, new trends in assisted reproduction have made marked changes in the practice of IVF as a whole, but are only starting to affect AI practices. An essential requirement for effective transfer is to decrease the trauma caused to the endocervix and endometrium during transfer using catheters. ${ }^{15}$ Very limited data are available regarding the effect of the catheter used for insemination on pregnancy and LBRs. A search of the literature revealed many prospective randomized controlled trials evaluating different embryo transfer catheters, but only a limited number evaluating different IUI catheters. This discrepancy is interesting as IUI is not only a much older, more widely available procedure than IVF, but that it is also more widely used in assisted reproduction than IVF.

The difference in IUI pregnancy rate could be attributed to different stimulation regimes, single or double IUI or selection of patients in the two groups as there was no randomization. Recent Cochrane does not support
Table 2: Results of the prospective study to evaluate the new intrauterine insemination cannula

\begin{tabular}{|c|c|c|c|}
\hline Parameter & $\begin{array}{l}\text { Study } \\
\text { group }\end{array}$ & $\begin{array}{l}\text { Control } \\
\text { group }\end{array}$ & $p^{*}$ \\
\hline Number of subjects $(n)$ & 45 & 45 & - \\
\hline Mean concentration $\left(10^{6} / \mathrm{ml}\right)$ & 59 & 62 & - \\
\hline Postwash motility (\%) & 90 & 90 & - \\
\hline IUI pregnancy rate, $\mathrm{n}(\%)$ & $10(22.2 \%)$ & $7(17.7 \%)$ & $<0.05$ \\
\hline Volume of seminal plasma & & & \\
\hline $\begin{array}{l}\text { leftover in the cannula }(\mathrm{ml}) \\
\text { Total sperm concentration }\end{array}$ & 0.02 & 1.4 & $<0.05$ \\
\hline Leftover in the cannula $\left(10^{6} / \mathrm{ml}\right)$ & 0.8 & 8.4 & $<0.05$ \\
\hline $\begin{array}{l}\text { Total motile sperm } \\
\text { concentration }\end{array}$ & & & \\
\hline leftover in the cannula (\%) & $>80$ & - & - \\
\hline
\end{tabular}

*Based on t-test $(95 \% \mathrm{CI})$, IUI: Intrauterine insemination

benefit of one type of catheter over another in IUI cycles (soft $v$ s firm). Moreover, use of stillet in IUI is debatable. However, the single important factor with the new design of IUI cannula is that it can transfer markedly higher number of good quality sperms, without contamination, as compared to the currently available cannula. Therefore, the increased pregnancy rate with the new design of IUI cannula has been attributed the better performance of the new cannula.

We have proposed a novel design for an insemination cannula which ensures complete emptying of semen thereby eliminating dead space and ensures sterility by removing the usage of air. Our study has found that the modification of the design increased pregnancy rates by nearly $5 \%$, a significant step toward achieving higher pregnancy rates. Other comparison statistics like residual seminal plasma volume $(1.4 \mathrm{ml}$ for control vs $0.2 \mathrm{ml}$ for study) and total sperm concentration leftover ( 8.4 million in control vs 0.8 million in study) also showed significant difference, demonstrate the superiority of using the new IUI cannula. This preliminary research warrants investigation by multicentric randomized clinical trials to draw definitive conclusions and establish the enhanced IUI pregnancy rates by the use of the new IUI cannula.

\section{REFERENCES}

1. Anon. What is intrauterine insemination (IUI) and how does it work? 2014, Dec 9. Available at: http://www.hfea.gov.uk/ IUI.html

2. Aboulghar H, Aboulghar M, Mansour R, Serour G, Amin Y, Al-Inany $\mathrm{H}$. A prospective controlled study of karyo-typing for 430 consecutive babies conceived through intracytoplasmic sperm injection. Fertil Steril 2001 Aug;76(2):249-253.

3. Cohen MR. Intrauterine insemination. Int J Fertil 1962;7: 235-240.

4. Pandian Z, Bhattacharya S, Vale L, Templeton A. In vitro fertilization for unexplained subfertility. Cochrane Database Syst Rev 2005;18,CD003357. 
5. Anon. What is intrauterine insemination (IUI)-chance of success 2014;Dec 9. Available at: http://www.hfea.gov.uk/ iui-success-rate.html

6. Ombelet W, Campo R, Bosmans E, Nijs M. Intrauterine insemination (IUI) as a first-line treatment in developing countries and methodological aspects that might influence IUI success. ESHRE Monogr 2008;1:64-72.

7. Campana A, Sakkas D, Stalberg A, Bianchi PG, Comte I, Pache $\mathrm{T}$, et al. Intrauterine insemination: evaluation of the results according to the women's age, sperm quality, total sperm count per insemination, and life table analysis. Hum Reprod 1996;11(4):732-736.

8. McGoven P, Quagliarello J, Arny M. Relationship of within patient semen variability to outcome of intrauterine insemination. Fertil Steril 1989 Jun;51(6):1019-1023.

9. Arny M, Quagliarello J. Semen quality before and after processing by a swim-up method: relationship of outcome of intrauterine insemination. Fertil Steril 1987 Oct;48(4):643-648.

10. Dorjpurev U, Kuwhara A, Yano Y, Taniguchi T, Yamamoto $Y$, Suto A, Tanaka Y, Matzuzaki T, Yasui T, Iraha M. Effect of semen characteristics on pregnancy rate following intrauterine insemination. J Med Investigation 2011;58(1-2):127-133.

11. Haim D, Leniaud L, Porcher R, Martin-Pont B, Wolf JP, Sifer $\mathrm{C}$. Evaluation prospective de l'impact des paramètres spermatiques sur le succès des inséminations intra-utérines. Gynecol Obstet Fertil 2009;37(3):229-235.

12. Youn JS, Cha SH, Yang KM, Kim JY, Koong MK, Kang IS, Song IO, Han SC. Predictive value of sperm motility charac- teristics assessed by computer-assisted sperm analysis in intrauterine insemination with superovulation in couples with unexplained infertility. Clin Exp Reprod Med 2011;38(1):47-52.

13. Jellad S, Basly M, Chibani M, Rachdi R. Predictive factors for intrauterine insemination success: analysis of semen parameters affecting outcome. Int J Urology 2014;12(1). Available at: https://ispub.com/IJU/12/1/20892

14. Hughes EG. Stimulated intrauterine insemination is not a natural choice for the treatment of unexplained subfertility: Effective treatment or not a natural choice? Hum Reprod 2003;18(5):912-914.

15. Abou-Setta AM, Al-Inany HG, Mansour RT, Serour GI, Aboulghar MA. Soft versus firm embryo transfer catheters for assisted reproduction: a systematic review and metaanalysis. Hum Reprod 2005;Nov20(11):3114-3121.

16. Kovacs GT. What factors are important for successful embryo transfer after in vitro fertilization? Hum Reprod 1999;14(3): 590-592.

17. Fancsovits P, Toth L, Takacs ZF, Murber A, Papp Z, UrbancsekJ. Early pronulcearbreakdown is a good indicator of embryo quality and viability. Fertil Steril 2005;84(4):881-887.

18. Proctor JG Jr, Boone WR. Economics of flexible vs rigid catheters for intrauterine insemination. Fertil Steril 2007; 88(3):749-750.

19. Abou-Setta AM, Mansour RT, Al-Inany HG, Aboulghar MA, Kamal A, Aboulghar MA, Serour G. Intrauterine insemination catheters for assisted reproduction: a systematic review and meta-analysis. Hum Reprod 2006;21(8):1961-1967. 